The seven donor hearts, in which ASD and TR had been produced beforehand, were heterotopically transplanted into the recipient's chest cavity. Two to three $\mathrm{mCi}$ of 111 In-antimyosin (AM) was injected intravenously 6-12 hours before excised. ${ }_{11}$ In-AM myocardial imaging was performed using scinticamera. T2 relaxation time $\left(\mathrm{T}_{2}\right)$ was calculated using NMR-CT. Then, histopathologic rejection score was also calculated and the severity of rejection was divided into mild, moderate and severe. Ill In-AM uptake was correlated with $\mathrm{T} 2$ and pathologic score. 111 In-AM uptake was higher in moderate and severe rejected myocardium, while $\mathrm{T} 2$ was prolonged from mild to severe rejected myocardium. Therefore, 111 Inantimyosin uptake was specific for myocyte necrosis with edema, or mononuclear infiltration compared with $\mathrm{T}_{2}$. This procedure allows to evaluate the therapeutic intervention upon cardiac rejection, leading to replacement of right ventricular endomyocardial biopsy.

$-332-$

A NEW METHOD FOR INFARCT SIZING -IMAGING OF ACUTE MYOCARDIAL INFARCTION WITH In-111 LABELED ANTIMYOSIN HEAVY CHAIN MONOCLONAL ANTIBODY-

Kazuhide Yamaoki, Yoshio Yazaki, Mituaki Isobe, Hidetsugu Tsuchimochi, Fumimaro Takaku, Mikiro Mori*, Jun-ichi Nishikawa , Masahiro Iio*

The Third Department of Medicine, and *The Department of Radiology, University of Tokyo, Tokyo, Japan.

In order to visualize and quantify the size of myocardial infarction (MI) noninvasively, we used monoclonal antibody (HMC50) specific for cardiac myosin heavy chain. HMC50 Fab fragment was labeled with indium(In)-111, via a bifunctional chelating agent, diethylene triamine penta-acetic acid. MI was produced by ligation of either left anterior descending ( 7 dogs) or circumflex coronary artery ( 21 dogs) in 28 dogs, weighing 7-8 $\mathrm{kg}$. In another dog right ventricular infarction was produced by right coranary ligation. At various intervals during 28 days after MI, single-photon emission computed tomography (SPECT) was performed using In-111 HMC50 Fab (0.4 $\mathrm{mCi} / \mathrm{kg}$ intravenously 22-24 hours before imaging) together with thallium (T1) $-201(0.1 \mathrm{mCi} / \mathrm{kg}, 0.5$ hour before imaging). In 8 cases technecium-99m pyrophosphate (TC-PYP: $1 \mathrm{mCi} / \mathrm{kg}, 3$ hours before imaging) scintigrams were also taken 2 days before In-111 scintigraphy. At necropsy the hearts were cut into $3 \mathrm{~mm}-\mathrm{s} l i c e s$. Pathologic infarct size (P-IS) determined by planimetry was compared with the size of hot spot of In-111 (In-IS). Infarct zone was clearly visualized by the In111 hot spot throughout 16 days after MI in all 25 cases. The location of the hot spot approximated to the actual infarct site and the cold spot of T1-201. In 3 cases 17-28 days after MI, no hot spot of In-111 was detectable in 3 cases. Uptake of Tc-pyp was scarecely visible beyond 7 days after coronary ligation. Unlike TC-PYP no bone uptake was noted in the images by In-111. Subendcardial ( 7 cases) and right ventricular infarction ( 1 case) were also visualized by In-111. During 8 days after left coronary ligation, In-IS correlated well with P-IS $(r=0.92: p<0.001$ in 8 cases 1 .

In conclusion SPECT with labeled HMC50 Fab was useful for infarct sizing as long as two weeks after acute myocardial infarction.
Chairman
$333-336$
$337-340$
K. Ishikawa, Osaka

S. Kimata, Tokyo

$-333-$

(Paper not submitted)

\section{$-334-$ \\ THE STUDY OF THE PROCESS OF HEART FAILURE AND THE RESTORATION IN EXPERIMENTAL ACUTE COR PULMONALE}

Toshiyuki Tanaka, Naomi Ono,

Kazuo Nakazima, Satoshi Okura,

Kiyotsugu Sekioka, Takeshi Nakano, Hideo Takezawa

The First Department of Internal Medicine of Mie University, Ttu, Mie

Many investigator have reported the hemodynamics of acute cor pulmonale, clinically and experimentally. However, the quantitative evaluation of the effect of coronary perfusion pressure and hypoxia on right ventricular contractility is still unknown. We simulated acute cor pulmonale in nine isolated canine hearts and investigated the process of heart failure and restoration. Afterload, preload of right ventricle was controlled with a computer-assisted volume control servosystem. And at the same time, coronary perfusion pressure (COPP) was also controlled with a computer by measured cardiac output. Decrease in contractility and right ventricular failure was defined as decrease in Emax and peak systolic pressure despite of increased afterload respectively. Withine the normal range of $\mathrm{PaO}_{2}$, the contractility of right ventricle decreased at COPP $64 \pm 7.1 \mathrm{mmHg}$ and right ventricle failed at COPP $51 \pm 5.1 \mathrm{mmHg}$. Withine the range of $\mathrm{PaO}_{2} 51 \pm 2.3 \mathrm{mmHg}$, the contractility of right ventricle decreased at COPP $72 \pm 3.8 \mathrm{~mm} \mathrm{Hg}$ and right ventricle failed at COPP $59 \pm 8.8 \mathrm{mmHg}$. As long as the COPP was normal, $90 \mathrm{mmHg}$, contractility of right ventricle did not decrease despite of hypoxia $\left(\mathrm{PaO}_{2} 51 \pm 2.3 \mathrm{mmHg}\right)$. The increase in systemic vascular resistance or administration of $\beta$-stimulant in the heart failure state restored the contractility of right ventricle, but increase in preload decreased the contractility of right ventricle.

$-335-$

CLINICAL SIGNIFICANCE OF LOW $\mathrm{T}_{3}$ SYNDROME IN ACUTE MYOCARDIAL INFARCTION

Yoshiki Tanaka, Toshiji Iwasaka, Hideki Onoyama, Mitsushige Nishikawa, Yoshihachirou Shiroyama, Izumi Yoshiyama, Akira Ichibangase, Yasuo Takayama, Mitsuo Inada

Second Department of Internal Medicine, Kansai Medical University

To assess the effect of thyroid hormone on left ventricular performance in acute myocardial infarction, serum $T_{4}$ and $T_{3}$ levels were serially measured by RIA 